\title{
Observational Study on the Recovery from Root Lodging at Flowering Time and Yield Reduction in Maize (Zea mays L.)
}

\author{
Yoon-Sup So ${ }^{1 *}$, Victor O. Adetimirin ${ }^{2}$, and Soon-Kwon $\mathrm{Kim}^{3}$ \\ ${ }^{1}$ Department of Crop Science, Chungbuk National University, Cheongju, Chungbuk, 361-763, Republic of Korea \\ ${ }^{2}$ Department of Agronomy, University of Ibadan, Ibadan, Nigeria \\ ${ }^{3}$ Handong Global University, Pohang, Gyeongbuk, 791-708, Republic of Korea
}

\begin{abstract}
On August 2, 1999, the typhoon "Olga" with the maximum wind speed of $19.2 \mathrm{~m} / \mathrm{s}$ hit the Korean peninsula and caused considerable damage to crops. Regional yield trials were at tasseling stage and were affected by the typhoon. After the storm, almost all the plants were root-lodged. This provided a rare chance to study the relationship between recovery from the lodging and reduction of grain yield in maize. Three check cultivars were examined for angle of lodging, grain yield, yield components, plant height and ear height. Six levels of leaning were classified. Plants leaning less than $10^{\circ}$ from the vertical were considered as non-lodged. Average reduction in grain yield across five levels of lodging was $29.2 \%$ with the mean of $46.1^{\circ}$. The reductions of three hybrids were $25 \%$ for Suwon 19, 39.5\% for Pioneer 3525 and $27.4 \%$ for DeKalb $689 . R^{2}$ values of grain yield on angles of lodging ranged from 0.33 for Suwon 19 to 0.51 for Pioneer 3525. The slope of the regression was lowest for Suwon 19 and highest for Pioneer 3525. Yield reduction from lodging was less severe for the locally bred Suwon 19. Reductions in yield components were also influenced by cultivars with Pioneer 3525 showing the most severe reduction. The yield components most severely affected by root lodging at the flowering time were ear length and number of kernels per row. The high reductions in yield and agronomic traits caused by root lodging justify the lodging resistance as a major breeding objective in Korea.
\end{abstract}

Keywords Maize, Root lodging, Yield reduction, Yield components, Typhoon

\section{INTRODUCTION}

Maize (Zea mays L.) is cultivated on approximately 100,000 ha in South Korea and 700,000 ha in North Korea respectively. Lodging, one of the destructive biotic stresses of maize, poses a limitation to maize cultivation in Korea, particularly for the silage production in South Korea and for the grain production in North Korea. In North Korea, breeding a short status of maize plants has been emphasized in spite of high negative correlations between the short plant and low yield reported due to root lodging (personal communication with Soon-Kwon Kim). Waxy corn production is less damaged by the root-lodging caused by the typhoon since the growers avoid the summer rainy season by early spring planting in covered facility and transplanting to the field later. Days to harvest are also relatively short for waxy corn than silage production.

Two types of lodging are recognized as root and stalk lodging. Root lodging is characterized by a leaning from the vertical of intact maize stalks (Fouéré et al. 1995), while stalk lodging manifests as breakage of the stalk below the uppermost ear. Of the two types of lodging, stalk lodging has by far the greater consequence for yield reduction, but it is less common compared to root lodging.

The occurrence of the two types of lodging depends on the time of heavy storms during plant growth and cultivars. Root lodging is often caused by corn rootworm (Diabrotica spp.), wind pressure, rain and subsequent wet soil or a combination of these factors (Melchinger et al. 1986; Hebert et al. 1992; Stamp and Kiel 1992; Fouéré et al. 1995). Also, agronomic practices such as higher planting density (Liu et al. 2012) and extravagant use of chemical

Received Jun 18, 2013; Revised Jun 23, 2013; Accepted Jun 26, 2013; Published June 30, 2013

*Comesponding author Yoon-Sup So, yoonsupso@cbnu.ac.kr, Tel: +82-43-261-2517, Fax: +82-43-273-2242 
fertilizers can predispose the crop to root lodging. Although lodging can occur at any stage of plant growth, yield reduction is most severe when it occurs around flowering time (Minami and Ujihara 1991). Carter and Hudelson (1982) and Minami and Ujihara (1991) obtained estimates of grain yield losses from experiments that simulated plant lodging caused by storms. Published information on the effects of root lodging on maize yield in Korea is not available. This may be due to the extreme difficulty of simulating uniform root lodging at field scale. In accessing abiotic stresses for crop improvement, it is also an important selection criterion to examine the degree of recovery from the damage as well as crop's ability to withstand the stresses.

In Korean peninsula, the main cause of root lodging is the seasonal typhoon that accompanies the high precipitation in summer (June - August). This was particularly happened in 1999. On August 2, 1999, the typhoon "Olga" with the maximum wind speed of $19.2 \mathrm{~m} / \mathrm{s}$ hit the peninsula and caused considerable damage to the crops including rice and maize. Regional maize yield trials were at tasseling stage and were also affected by the typhoon. After the storm, almost all the plants were root-lodged, flat on the ground. This provided a rare chance to study the relationship between recovery from the root lodging and reduction of grain yield in maize. This report provides the result from the observational study given by the typhoon "Olga".

\section{MATERIALS AND METHODS}

Data for this study were collected on three commercial hybrid cultivars used as checks in maize yield trials carried out at the experiment station of Kyungpook National University (lat. 36 $7^{\circ}$; long. 128 $37^{\circ} \mathrm{E}$ ) at Kunwi, in 1999. A total of 25 trials were involved, each consisting of 40 experimental hybrids. There were two check hybrids per trial as Suwon 19 and Pioneer 3535 or DeKalb 689. The last two hybrids are commercial US single cross hybrids marketed in Korea while Suwon 19 is a South Korea hybrid developed in 1970s (Kim et al. 1978). Experimental design for each of the trial was arranged in a randomized complete block with three replications. Plots consisted of single rows spaced $0.7 \mathrm{~m}$ apart with $2 \mathrm{~m}$ row length. Hill spacing was $0.25 \mathrm{~m}$ with two plants maintained per hill, giving a high population density of 114,286 plants per hectare. The alley lengths of each range (planting block) were $0.75 \mathrm{~m}$ for big and $0.5 \mathrm{~m}$ for small. Nitrogen was applied at the rate of 180 $\mathrm{kg} /$ ha with half at planting time. Phosphorus and potassium were each applied as base at the rate of $150 \mathrm{~kg} / \mathrm{ha}$.

On August 2, the typhoon 'Olga' landed on the Korean peninsula. It was about 65 day after planting maize and was around flowering time. All plants were exposed to maximum wind speed of $19.5 \mathrm{~m} / \mathrm{s}$ accompanied by $43 \mathrm{~mm}$ precipitation, resulting in severe root lodging. The occurrence of the typhoon provided an opportunity to quantify yield losses of maize due to root lodging in Korea.

At harvesting time, angle of lodging was determined at $10 \mathrm{~cm}$ below the first ear for individual plants of three hybrids in all 25 trials. Other agronomic traits determined included plant height, ear height, ear weight, ear length, ear diameter, ear circumference, number of kernels per row, number of kernel rows, cob weight, cob diameter, 100kernerl weight and grain moisture content. One hundredkernel weight and grain yields were adjusted to $15 \%$ moisture content.

Plants were grouped into one of six levels (angle) of lodging. Plants with less than $10^{\circ}$ leaning angle from the vertical were considered as non-lodged. Data were analyzed with SAS program (SAS Inst. Cary, NC). Grain yield was regressed on angle of lodging. Regression was carried out of each hybrid and also across hybrids. Relationships among grain yield and agronomic traits were investigated by correlation analyses.

\section{RESULTS AND DISCUSSION}

The maximum wind speed of $19.2 \mathrm{~m} / \mathrm{s}$ to which the trials in this study were exposed was very high. Human movement against wind direction is considered not possible beyond $17.2 \mathrm{~m} / \mathrm{s}$, and at such wind speed small branches of tree are easily broken according to the storm description developed by Korea weather service.

The drawbacks of the present study are as follows: 1) the data reported here include data from one year only because 
similar lodging did not occur in 2000, and 2) data collections were made from not pre-designed experiment with three cultivars. The authors captured a historical opportunity of root lodging damage that occurred in a large set of yield trials of maize. Only three cultivars were selected to study lodging because these hybrids are highly uniform single crosses and also are popular commercial hybrids marketed in South Korea.

Rainstorms do not usually affect plots within trials uniformly (Fouéré et al. 1995). However, collection of data from standard check cultivars in the 25 trials provided various environmental conditions that offered random effect assessment of the lodging to grain yield and agronomic traits. The hybrids used are single crosses with high uniformity that adjust to minimize any biases and misinterpretation of data. In addition, no single data is available in this aspect of information.

Although numbers of plants sampled were different, the

Table 1. Mean $( \pm \mathrm{sd})$ values of agronomic traits and grain yields of three maize hybrids at varying angles of lodging.

\begin{tabular}{|c|c|c|c|c|c|c|}
\hline \multirow{2}{*}{ Hybrid } & \multirow{2}{*}{ No. of plant } & \multicolumn{2}{|c|}{ Angle of lodging } & \multirow{2}{*}{ Plant height } & \multirow{2}{*}{ Ear height } & \multirow{2}{*}{ Grain yield } \\
\hline & & Range & Mean & & & \\
\hline & & \multicolumn{2}{|c|}{ - - - } & \multicolumn{2}{|c|}{$\mathrm{cm}$} & $\mathrm{g} /$ plant \\
\hline \multirow[t]{7}{*}{ Suwon 19} & 8 & $<10$ & $5.5 \pm 3.96$ & $257.6 \pm 27.29$ & $136.8 \pm 12.12$ & $317.1 \pm 30.17$ \\
\hline & 43 & $10 \sim 24$ & $17.6 \pm 4.98$ & $259.5 \pm 16.19$ & $133.9 \pm 11.01$ & $284.4 \pm 32.55$ \\
\hline & 37 & $25 \sim 39$ & $32.6 \pm 4.48$ & $262.5 \pm 23.15$ & $137.1 \pm 13.76$ & $245.1 \pm 40.99$ \\
\hline & 21 & $40 \sim 54$ & $46.4 \pm 4.99$ & $267.2 \pm 16.69$ & $141.0 \pm 11.01$ & $257.7 \pm 34.09$ \\
\hline & 8 & $55 \sim 69$ & $56.0 \pm 1.31$ & $268.8 \pm 13.82$ & $142.5 \pm 12.25$ & $231.7 \pm 25.81$ \\
\hline & 4 & $70 \sim 90$ & $72.0 \pm 0$ & $267.5 \pm 2.89$ & $133.5 \pm 7.51$ & $169.9 \pm 26.28$ \\
\hline & & Mean $^{\text {z) }}$ & $30.4 \pm 16.35$ & $262.5 \pm 19.13$ & $136.9 \pm 12.11$ & $262.7 \pm 44.67$ \\
\hline \multirow[t]{7}{*}{ P3525 } & 10 & $<10$ & $3.5 \pm 1.51$ & $276.5 \pm 7.58$ & $154.3 \pm 11.96$ & $338.9 \pm 40.20$ \\
\hline & 21 & $10 \sim 24$ & $17.2 \pm 4.71$ & $265.2 \pm 27.88$ & $139.7 \pm 14.60$ & $278.5 \pm 43.10$ \\
\hline & 18 & $25 \sim 39$ & $29.9 \pm 3.68$ & $249.8 \pm 22.00$ & $139.2 \pm 15.48$ & $253.1 \pm 48.86$ \\
\hline & 4 & $40 \sim 54$ & $42.3 \pm 1.71$ & $228.8 \pm 15.48$ & $126.8 \pm 10.28$ & $182.4 \pm 69.48$ \\
\hline & 1 & $55 \sim 69$ & 56.0 & 275.0 & 160.0 & 106.0 \\
\hline & & $70 \sim 90$ & & & & \\
\hline & & Mean & $21.5 \pm 12.67$ & $259.6 \pm 25.44$ & $141.7 \pm 15.63$ & $270.9 \pm 64.21$ \\
\hline \multirow[t]{7}{*}{ DK689 } & 10 & $<10$ & $3.8 \pm 2.70$ & $248.0 \pm 13.35$ & $141.7 \pm 15.57$ & $357.3 \pm 70.07$ \\
\hline & 16 & $10 \sim 24$ & $18.1 \pm 4.93$ & $252.8 \pm 18.69$ & $143.5 \pm 10.37$ & $332.0 \pm 73.22$ \\
\hline & 13 & $25 \sim 39$ & $29.8 \pm 4.04$ & $248.2 \pm 14.94$ & $141.8 \pm 8.92$ & $285.7 \pm 41.51$ \\
\hline & 5 & $40 \sim 54$ & $47.2 \pm 4.21$ & $264.8 \pm 21.22$ & $150.0 \pm 20.54$ & $296.4 \pm 15.70$ \\
\hline & 3 & $55 \sim 69$ & $65.3 \pm 2.52$ & $234.0 \pm 3.61$ & $142.3 \pm 6.81$ & $181.1 \pm 20.54$ \\
\hline & 6 & $70 \sim 90$ & $81.5 \pm 5.01$ & $260.8 \pm 27.82$ & $161.0 \pm 15.94$ & $201.3 \pm 34.20$ \\
\hline & & Mean & $30.9 \pm 24.45$ & $251.7 \pm 18.52$ & $145.3 \pm 13.69$ & $298.7 \pm 76.67$ \\
\hline \multirow[t]{7}{*}{ Across } & 28 & $<10$ & $4.2 \pm 2.83$ & $260.9 \pm 20.65$ & $144.8 \pm 14.93$ & $339.3 \pm 51.74$ \\
\hline & 80 & $10 \sim 24$ & $17.4 \pm 4.97$ & $260.1 \pm 20.54$ & $137.6 \pm 12.39$ & $292.6 \pm 49.02$ \\
\hline & 68 & $25 \sim 39$ & $31.2 \pm 4.48$ & $256.5 \pm 21.96$ & $138.4 \pm 13.28$ & $256.5 \pm 45.61$ \\
\hline & 30 & $40 \sim 54$ & $46.0 \pm 4.72$ & $261.7 \pm 21.28$ & $140.6 \pm 13.94$ & $254.1 \pm 48.61$ \\
\hline & 12 & $55 \sim 69$ & $58.3 \pm 4.48$ & $260.6 \pm 19.60$ & $143.9 \pm 11.38$ & $208.6 \pm 45.33$ \\
\hline & 10 & $70 \sim 90$ & $77.7 \pm 6.17$ & $263.5 \pm 21.09$ & $150.0 \pm 19.01$ & $188.8 \pm 33.82$ \\
\hline & & Mean & $28.6 \pm 18.20$ & $259.3 \pm 21.03$ & $140.0 \pm 13.78$ & $273.0 \pm 59.81$ \\
\hline
\end{tabular}

\footnotetext{
${ }^{\mathrm{z})}$ Means of traits across lodging angles exclude the $<10^{\circ}$ range; plants in this category were considered as non-lodged.
} 
three hybrids showed similar modal range for angle of lodging $\left(10^{\circ}-24^{\circ}\right)$ (Table 1). Grain yields of non-lodged plants $\left(<10^{\circ}\right)$ averaged as increasing order of $317.1 \mathrm{~g} /$ plant for Suwon 19, $338.9 \mathrm{~g} /$ plant for Pioneer 3525 and 357.3 $\mathrm{g} /$ plant for DeKalb 689 (Table 1), indicating relative yield advantages of recently bred two introduced hybrids. Across five levels of lodging (excluding the non-lodged level, $>10^{\circ}$ ), reduction in grain yield was $25.0 \%$ for Suwon
19 (79.3 g/plant), 39.5\% for Pioneer 3525 (133.9 g/plant) and 27.4\% for DeKalb 689 (98.0 g/plant). These values were considerably higher than the $15.8 \%$ reported by Minami and Ujihara (1991), but comparable to the 25\% reported by Zuber and Kang (1978) in USA.

The results clearly indicate the importance of the data presented in this study. Grain yield reduction due to lodging is known to result from a direct effect of lower dry

Table 2. Mean $( \pm \mathrm{sd})$ values of yield components of three maize hybrids at varying angles of lodging.

\begin{tabular}{|c|c|c|c|c|c|c|c|c|c|c|}
\hline \multirow{2}{*}{ Hybrid } & \multirow{2}{*}{$\begin{array}{c}\text { No. of } \\
\text { plant }\end{array}$} & \multicolumn{2}{|c|}{ Angle of lodging } & \multirow{2}{*}{ Ear length } & \multirow{2}{*}{$\begin{array}{c}\text { Ear } \\
\text { diameter }\end{array}$} & \multirow{2}{*}{$\begin{array}{c}\text { Ear } \\
\text { circumference }\end{array}$} & \multirow{2}{*}{$\begin{array}{c}\text { Cob } \\
\text { diameter }\end{array}$} & \multirow{2}{*}{$\begin{array}{c}\text { 100-kernel } \\
\text { weight }\end{array}$} & \multirow{2}{*}{$\begin{array}{l}\text { No. of } \\
\text { kernels } \\
\text { per row }\end{array}$} & \multirow{2}{*}{$\begin{array}{c}\text { No. of } \\
\text { kernel } \\
\text { rows }\end{array}$} \\
\hline & & Range & Mean & & & & & & & \\
\hline & & \multicolumn{2}{|c|}{ - - $\circ \circ-\cdots$} & \multicolumn{6}{|c|}{ - - - - - $\mathrm{mm}--\cdots-\cdots-\mathrm{g}$} & \\
\hline \multirow{7}{*}{ Suwon 19} & 8 & $<10$ & $5.5 \pm 3.96$ & $162.4 \pm 59.73$ & $47.1 \pm 1.36$ & $158.0 \pm 7.37$ & $25.5 \pm 3.06$ & $38.0 \pm 2.35$ & $42.6 \pm 2.97$ & $13.5 \pm 1.41$ \\
\hline & 43 & $10 \sim 24$ & $17.6 \pm 4.49$ & $175.4 \pm 16.48$ & $45.7 \pm 1.71$ & $148.5 \pm 5.65$ & $24.0 \pm 1.75$ & $37.0 \pm 4.36$ & $39.3 \pm 3.70$ & $13.6 \pm 1.78$ \\
\hline & 37 & $25 \sim 39$ & $32.6 \pm 4.48$ & $154.9 \pm 22.91$ & $44.5 \pm 2.16$ & $146.0 \pm 7.61$ & $23.8 \pm 2.11$ & $35.6 \pm 3.63$ & $36.5 \pm 7.22$ & $13.7 \pm 1.43$ \\
\hline & 21 & $40 \sim 54$ & $46.4 \pm 4.99$ & $170.6 \pm 16.39$ & $44.9 \pm 3.45$ & $149.1 \pm 7.02$ & $24.0 \pm 2.12$ & $34.0 \pm 2.93$ & $40.0 \pm 3.76$ & $13.7 \pm 1.53$ \\
\hline & 8 & $55 \sim 69$ & $56.0 \pm 1.31$ & $168.0 \pm 13.42$ & $44.0 \pm 0.76$ & $141.0 \pm 4.72$ & $24.3 \pm 2.76$ & $32.9 \pm 2.07$ & $39.3 \pm 4.10$ & $13.0 \pm 1.07$ \\
\hline & 4 & 70 90 & $72.0 \pm 0$ & $130.0 \pm 10.39$ & $44.5 \pm 0.58$ & $147,0 \pm 1.15$ & $25.5 \pm 2.89$ & $38.0 \pm 3.54$ & $26.5 \pm 4.04$ & $13.0 \pm 1.15$ \\
\hline & & Mean $^{\text {z) }}$ & $30.7 \pm 16.35$ & $165.4 \pm 25.23$ & $45.1 \pm 2.27$ & $147.9 \pm 7.27$ & $24.1 \pm 2.13$ & $35.9 \pm 3.88$ & $38.4 \pm 5.71$ & $13.6 \pm 1.54$ \\
\hline \multirow{7}{*}{ P3525 } & 10 & $<10$ & $3.5 \pm 1.51$ & $180.1 \pm 36.02$ & $46.8 \pm 1.48$ & $153.6 \pm 5.78$ & $24.9 \pm 1.45$ & $35.3 \pm 2.68$ & $45.0 \pm 2.71$ & $15.0 \pm 1.70$ \\
\hline & 21 & $10 \sim 24$ & $17.2 \pm 4.71$ & $162.6 \pm 25.82$ & $45.7 \pm 2.61$ & $150 \pm 7.00$ & $24.3 \pm 1.59$ & $33.4 \pm 2.75$ & $40.6 \pm 4.89$ & $15.4 \pm 1.69$ \\
\hline & 18 & $25 \sim 39$ & $29.9 \pm 3.68$ & $161.1 \pm 20.44$ & $45.3 \pm 1.46$ & $148.6 \pm 5.25$ & $24.3 \pm 1.03$ & $31.3 \pm 3.13$ & $38.9 \pm 4.87$ & $15.2 \pm 1.56$ \\
\hline & 4 & $40 \sim 54$ & $42.3 \pm 1.71$ & $142.8 \pm 31.10$ & $42.5 \pm 3.87$ & $139.0 \pm 10.52$ & $23.0 \pm 1.41$ & $29.8 \pm 5.61$ & $34.5 \pm 8.66$ & $13.5 \pm 1.91$ \\
\hline & 1 & $55 \sim 69$ & 56.0 & 90.0 & 43.0 & 138.0 & 22.0 & 31.9 & 21.0 & 17.0 \\
\hline & & 70 90 & & & & & & & & \\
\hline & & Mean & $21.5 \pm 12.67$ & $162.5 \pm 29.13$ & $45.5 \pm 2.37$ & $149.1 \pm 7.32$ & $24.3 \pm 1.43$ & $32.8 \pm 3.52$ & $40.0 \pm 6.04$ & $15.2 \pm 1.69$ \\
\hline \multirow{7}{*}{ DK689 } & 10 & $<10$ & $3.8 \pm 2.70$ & $186.8 \pm 19.81$ & $47.3 \pm 3.80$ & $154.6 \pm 7.50$ & $27.0 \pm 1.70$ & $33.8 \pm 2.90$ & $45.2 \pm 3.39$ & $15.2 \pm 1.03$ \\
\hline & 16 & $10 \sim 24$ & $18.1 \pm 4.73$ & $176.1 \pm 22.46$ & $47.6 \pm 2.55$ & $154.4 \pm 9.43$ & $27.1 \pm 1.31$ & $32.1 \pm 3.78$ & $43.1 \pm 5.47$ & $15.4 \pm 1.20$ \\
\hline & 13 & $25 \sim 39$ & $29.8 \pm 4.04$ & $176.2 \pm 24.82$ & $45.6 \pm 2.26$ & $148.5 \pm 7.11$ & $25.6 \pm 1.33$ & $31.1 \pm 3.13$ & $43.2 \pm 4.93$ & $14.5 \pm 2.03$ \\
\hline & 5 & $40 \sim 54$ & $47.2 \pm 4.21$ & $172.8 \pm 9.20$ & $47.0 \pm 2.00$ & $152.8 \pm 5.54$ & $26.2 \pm 1.30$ & $33.6 \pm 2.83$ & $42.8 \pm 1.79$ & $15.2 \pm 1.10$ \\
\hline & 3 & $55 \sim 69$ & $65.3 \pm 2.52$ & $138.0 \pm 31.00$ & $44.3 \pm 2.31$ & $143.3 \pm 5.77$ & $24.0 \pm 1.00$ & $25.0 \pm 6.12$ & $36.0 \pm 9.17$ & $15.3 \pm 2.31$ \\
\hline & 6 & $70 \sim 90$ & $81.5 \pm 5.01$ & $118.2 \pm 39.19$ & $44.3 \pm 1.86$ & $145.3 \pm 4.23$ & $24.3 \pm 1.37$ & $32.7 \pm 4.72$ & $30.8 \pm 4.58$ & $15.2 \pm 2.99$ \\
\hline & & Mean & $30.9 \pm 24.45$ & $169.1 \pm 31.48$ & $46.5 \pm 2.82$ & $151.2 \pm 8.20$ & $26.2 \pm 1.70$ & $32.0 \pm 4.01$ & $41.7 \pm 6.39$ & $15.1 \pm 1.69$ \\
\hline \multirow{7}{*}{ Across } & 28 & $<10$ & $4.2 \pm 2.83$ & $177.4 \pm 39.88$ & $47.1 \pm 2.46$ & $155.2 \pm 6.88$ & $25.8 \pm 2.24$ & $35.5 \pm 3.08$ & $44.4 \pm 3.14$ & $14.6 \pm 1.54$ \\
\hline & 80 & $10 \sim 24$ & $17.4 \pm 4.97$ & $172.4 \pm 20.94$ & $46.1 \pm 2.24$ & $150.3 \pm 7.17$ & $24.6 \pm 2.02$ & $35.1 \pm 4.37$ & $40.5 \pm 4.60$ & $14.4 \pm 1.87$ \\
\hline & 68 & $25 \sim 39$ & $31.2 \pm 4.48$ & $161.5 \pm 24.03$ & $45.0 \pm 2.05$ & $147.3 \pm 6.93$ & $24.3 \pm 1.85$ & $33.7 \pm 4.07$ & $38.5 \pm 6.62$ & $14.3 \pm 1.67$ \\
\hline & 30 & $40 \sim 54$ & $46.0 \pm 4.72$ & $167.3 \pm 19.83$ & $44.9 \pm 3.44$ & $148.4 \pm 8.10$ & $24.2 \pm 2.11$ & $33.4 \pm 3.51$ & $39.8 \pm 4.84$ & $13.9 \pm 1.58$ \\
\hline & 12 & $55 \sim 69$ & $58.3 \pm 4.48$ & $154.0 \pm 29.56$ & $44.0 \pm 1.21$ & $141.3 \pm 4.74$ & $24.0 \pm 2.34$ & $30.8 \pm 4.69$ & $36.9 \pm 7.29$ & $13.9 \pm 1.93$ \\
\hline & 10 & 70 90 & $77.7 \pm 6.17$ & $122.9 \pm 30.44$ & $44.4 \pm 1.43$ & $146.0 \pm 3.33$ & $24.8 \pm 2.04$ & $34.8 \pm 4.93$ & $29.1 \pm 4.70$ & $14.3 \pm 2.58$ \\
\hline & & Mean & $28.6 \pm 18.20$ & $165.6 \pm 27.69$ & $45.5 \pm 2.48$ & $149.0 \pm 7.59$ & $24.6 \pm 2.07$ & $34.2 \pm 4.20$ & $39.5 \pm 6.08$ & $14.3 \pm 1.78$ \\
\hline
\end{tabular}

\footnotetext{
${ }^{2)}$ Means of traits across lodging angles exclude the $<10^{\circ}$ range; plants in this category were considered non-lodged.
} 
matter accumulation and an indirect effect associated with harvesting losses (Carter \& Hudelson 1978; Minami \& Ujihara 1991).

Among the yield components measured, number of kernel rows of cultivars was the least affected by the lodging, while number of kernels per row and ear length were most affected, implying that the lodging occurred at the late stage of plant development. The kernel rows are decided at the early stage of the plant development. This trend was more evident at the highest level of lodging. Reduction in kernels per row across lodging levels was $14.8 \%$ for Suwon 19, 24.9\% for Pioneer 3525 and $13.2 \%$ for DeKalb 689, while reduction in ear length was $1.6 \%$ for Suwon 19, 22.5\% for P3525 and $16.3 \%$ for DK 689 . Although the effect of root lodging on ear length was not as great in Suwon 19 as the other two cultivars, it also suffered

Table 3. Correlation coefficients among agronomic traits, grain yield and yield components of maize hybrids.

\begin{tabular}{|c|c|c|c|c|c|c|c|c|c|c|c|}
\hline Traits & $\begin{array}{c}\text { Angle of } \\
\text { lodging } \\
\text { (AL) }\end{array}$ & $\begin{array}{c}\text { Plant } \\
\text { height } \\
(\mathrm{PH})\end{array}$ & $\begin{array}{c}\text { Ear height } \\
(\mathrm{EH})\end{array}$ & $\begin{array}{l}\text { Ear length } \\
\quad(\mathrm{EL})\end{array}$ & $\begin{array}{c}\text { Ear } \\
\text { diameter } \\
(E D)\end{array}$ & $\begin{array}{l}\text { No. of } \\
\text { kernels } \\
\text { per row } \\
(\mathrm{NKR})\end{array}$ & $\begin{array}{c}\text { No. of } \\
\text { kernel } \\
\text { rows } \\
\text { (KR) }\end{array}$ & $\begin{array}{c}\text { Ear } \\
\text { circumfer } \\
\text { ence } \\
\text { (CIR) }\end{array}$ & $\begin{array}{c}\text { Cob } \\
\text { diameter } \\
(\mathrm{CD})\end{array}$ & $\begin{array}{c}\text { 100-kerne } \\
1 \text { weight } \\
(\mathrm{KW})\end{array}$ & $\begin{array}{l}\text { Grain } \\
\text { yield } \\
(\mathrm{GY})\end{array}$ \\
\hline $\mathrm{AL}$ & & 0.21 & $0.18^{*}$ & -0.17 & $-0.26 * *$ & $-0.24 * *$ & -0.06 & $-0.28 * *$ & -0.05 & $-0.26^{* *}$ & $-0.58 * *$ \\
\hline $\mathrm{PH}$ & 0.03 & & $0.69 * *$ & 0.14 & 0.06 & 0.16 & 0.17 & 0.08 & -0.23 & -0.03 & 0.13 \\
\hline $\mathrm{EH}$ & 0.11 & $0.57 * *$ & & 0.08 & 0.14 & 0.16 & 0.28 & 0.11 & -0.05 & $-0.23 *$ & 0.07 \\
\hline EL & $-0.35 * *$ & 0.11 & -0.02 & & $0.18^{*}$ & $0.65^{* *}$ & -0.13 & $0.19 *$ & -0.09 & -0.02 & $0.58^{* *}$ \\
\hline ED & $-0.33 * *$ & 0.10 & 0.12 & $0.27 * *$ & & $0.26^{* *}$ & 0.11 & $0.59 * *$ & $0.2^{*}$ & $0.33 * *$ & 0.44 \\
\hline NKR & $-0.41 * *$ & 0.09 & 0.10 & $0.67 * *$ & $0.36 * *$ & & $-0.27 * *$ & 0.12 & $-0.19 *$ & -0.11 & $0.72 * *$ \\
\hline $\mathrm{KR}$ & -0.09 & 0.08 & $0.21 * *$ & -0.10 & $0.32 * *$ & -0.08 & & $0.24 * *$ & $0.34 * *$ & $-0.43^{* *}$ & -0.09 \\
\hline CIR & $-0.36^{* *}$ & 0.18 & 0.12 & $0.28 * *$ & $0.70 * *$ & $0.28 * *$ & $0.37 * *$ & & $0.31 * *$ & $0.41 * *$ & $0.5^{* *}$ \\
\hline $\mathrm{CD}$ & $-0.19 * *$ & $-0.15^{*}$ & 0.02 & 0.07 & $0.39 * *$ & 0.10 & $0.43 * *$ & $0.45 * *$ & & -0.03 & -0.06 \\
\hline KW & $-0.20 * *$ & $0.22 * *$ & -0.11 & 0.11 & $0.21 * *$ & -0.04 & $-0.49 * *$ & $0.3 * *$ & -0.13 & & $0.26^{* *}$ \\
\hline GY & $-0.60 * *$ & $0.19 * *$ & 0.09 & $0.61 * *$ & $0.59 * *$ & $0.72 * *$ & 0.11 & $0.64 * *$ & $0.28 * *$ & $0.26 * *$ & \\
\hline
\end{tabular}

Above diagonal: Suwon 19, below diagonal : across hybrids

Table 4. Correlation coefficients among agronomic traits, grain yield and yield components of maize hybrids.

\begin{tabular}{|c|c|c|c|c|c|c|c|c|c|c|c|}
\hline Traits & $\begin{array}{c}\text { Angle of } \\
\text { lodging } \\
\text { (AL) }\end{array}$ & $\begin{array}{c}\text { Plant } \\
\text { height } \\
(\mathrm{PH})\end{array}$ & $\begin{array}{c}\text { Ear height } \\
(\mathrm{EH})\end{array}$ & $\begin{array}{l}\text { Ear length } \\
\text { (EL) }\end{array}$ & $\begin{array}{c}\text { Ear } \\
\text { diameter } \\
(\mathrm{ED})\end{array}$ & $\begin{array}{l}\text { No. of } \\
\text { kernels } \\
\text { per row } \\
\text { (NKR) }\end{array}$ & $\begin{array}{c}\text { No. of } \\
\text { kernel } \\
\text { rows } \\
\text { (KR) }\end{array}$ & $\begin{array}{c}\text { Ear } \\
\text { circumfer } \\
\text { ence } \\
\text { (CIR) }\end{array}$ & $\begin{array}{c}\text { Cob } \\
\text { diameter } \\
(\mathrm{CD})\end{array}$ & $\begin{array}{c}\text { 100-kerne } \\
\text { 1 weight } \\
(\mathrm{KW})\end{array}$ & $\begin{array}{l}\text { Grain } \\
\text { yield } \\
(\mathrm{GY})\end{array}$ \\
\hline $\mathrm{AL}$ & & $-0.45^{* *}$ & $-0.29 *$ & $-0.39 * *$ & $-0.49 * *$ & $-0.59 * *$ & -0.08 & $-0.54 * *$ & $-0.39 * *$ & $-0.47 * *$ & $-0.72 * *$ \\
\hline $\mathrm{PH}$ & 0.10 & & $0.73 * *$ & 0.08 & $0.35 * *$ & 0.24 & 0.19 & $0.48 * *$ & $0.29 *$ & $0.56^{* *}$ & $0.49 * *$ \\
\hline $\mathrm{EH}$ & $0.32 *$ & $0.46^{* *}$ & & 0.01 & $0.39 *$ & 0.15 & 0.07 & 0.26 & 0.09 & $0.43 * *$ & 0.27 \\
\hline EL & $-0.63 * *$ & 0.16 & -0.25 & & 0.14 & $0.71 * *$ & -0.23 & 0.20 & 0.14 & $0.35^{* *}$ & $0.59 * *$ \\
\hline ED & $-0.40 * *$ & 0.09 & -0.30 & $0.50^{* *}$ & & 0.32 & $0.46^{* *}$ & $0.81^{* *}$ & $0.54 * *$ & $0.38^{* *}$ & $0.58 * *$ \\
\hline NKR & $-0.63 * *$ & -0.01 & -0.26 & $0.77 * *$ & $0.46^{* *}$ & & $-0.27 *$ & $0.38 * *$ & 0.26 & $0.49 * *$ & $0.79 * *$ \\
\hline $\mathrm{KR}$ & 0.01 & 0.16 & 0.11 & 0.01 & $0.41 * *$ & 0.02 & & $0.52 * *$ & $0.58^{* *}$ & -0.25 & 0.07 \\
\hline CIR & $-0.43 * *$ & 0.24 & -0.17 & $0.48^{* *}$ & $0.76^{* *}$ & $0.38^{* *}$ & $0.40 * *$ & & $0.71 * *$ & $0.48 * *$ & $0.73 * *$ \\
\hline $\mathrm{CD}$ & $-0.56^{* *}$ & -0.67 & $-0.35^{* *}$ & $0.32 *$ & $0.55^{* *}$ & $0.36^{* *}$ & $0.38 * *$ & $0.52 * *$ & & 0.05 & 0.43 \\
\hline KW & -0.23 & 0.13 & -0.01 & 0.21 & $0.28^{*}$ & 0.03 & $-0.39 * *$ & $0.37^{* *}$ & 0.09 & & $0.66^{* *}$ \\
\hline GY & $-0.69^{* *}$ & 0.18 & -0.22 & $0.69^{* *}$ & $0.71 * *$ & $0.66^{* *}$ & 0.18 & $0.74 * *$ & $0.53 * *$ & $0.40^{* *}$ & \\
\hline
\end{tabular}

Above diagonal: P3525, below diagonal : DK 689 
a considerable loss in kernels per row. Pair-wise comparisons on the degree of reduction in the two traits among the hybrids indicate that hybrids responded differently to the same level of damage. However, the trend of simultaneous reductions of these two traits by lodging was understandable because number of kernels per row determines ear length. Both traits were highly correlated $(P<0.01)$ for all three hybrids (Table $3 \& 4$ ).

Reductions ranged from 4.0 to $6.0 \%$ for cob diameter and 6.6 to $10.5 \%$ for $100-$ kernel weight. In addition to ear length, severity of reduction for these three traits were Pioneer $3525>$ DeKalb $689>$ Suwon 19. Ranks of cultivars for reduction severity were Pioneer $3525>$ Suwon $19>$ DeKalb 689 for ear diameter (range $=3.2-6.0$ ) and number of kernels per row (range $=13.2 \sim 24.9)$. Reduction in ear diameter was more severe for Suwon 19 than Pioneer 3525 and DeKalb 689.

Angle of lodging was significantly correlated $(P<0.01)$ to grain yield. The correlation $(r)$ between angle of lodging and grain yield was -0.58 for Suwon $19,-0.72$ for Pioneer 3525 and -0.69 for DeKalb 689 (Tables 3 and 4). Between $33 \%$ (Suwon 19) and 51\% (Pioneer 3525) of the variation in yield of the hybrids was accounted for by lodging (Fig. 1).

Suwon 19 showed a lower intercept value than Pioneer 3525 and DeKalb 689 while it had the lowest $b$ value of -1.58 , which was less than half the $b$ value for pioneer 3525 . Although Suwon 19 yielded less than the two introduced hybrids it showed less reduction in percent yield and agronomic traits by the lodging, indicating stability of the locally bred hybrid.

High reductions in grain yield and agronomic traits obtained in this study indicate that root lodging by typhoons could affect maize crop significantly on the Korean peninsula. The development of lodging-resistant cultivars should, therefore, be a major breeding objective. In addition the authors observed severe effect of root lodging on ear formation and development in Pyongchang Research Station in Kangwon Province in 1999 (Kim SK and Min HG et al. unpublished). Further studies need to be done on the effect of lodging that might occur at different stages of plant development. The results of maize yield reduction by root lodging in this observational study could provide an insight on determining the threshold value for rood lodging and yield reduction.
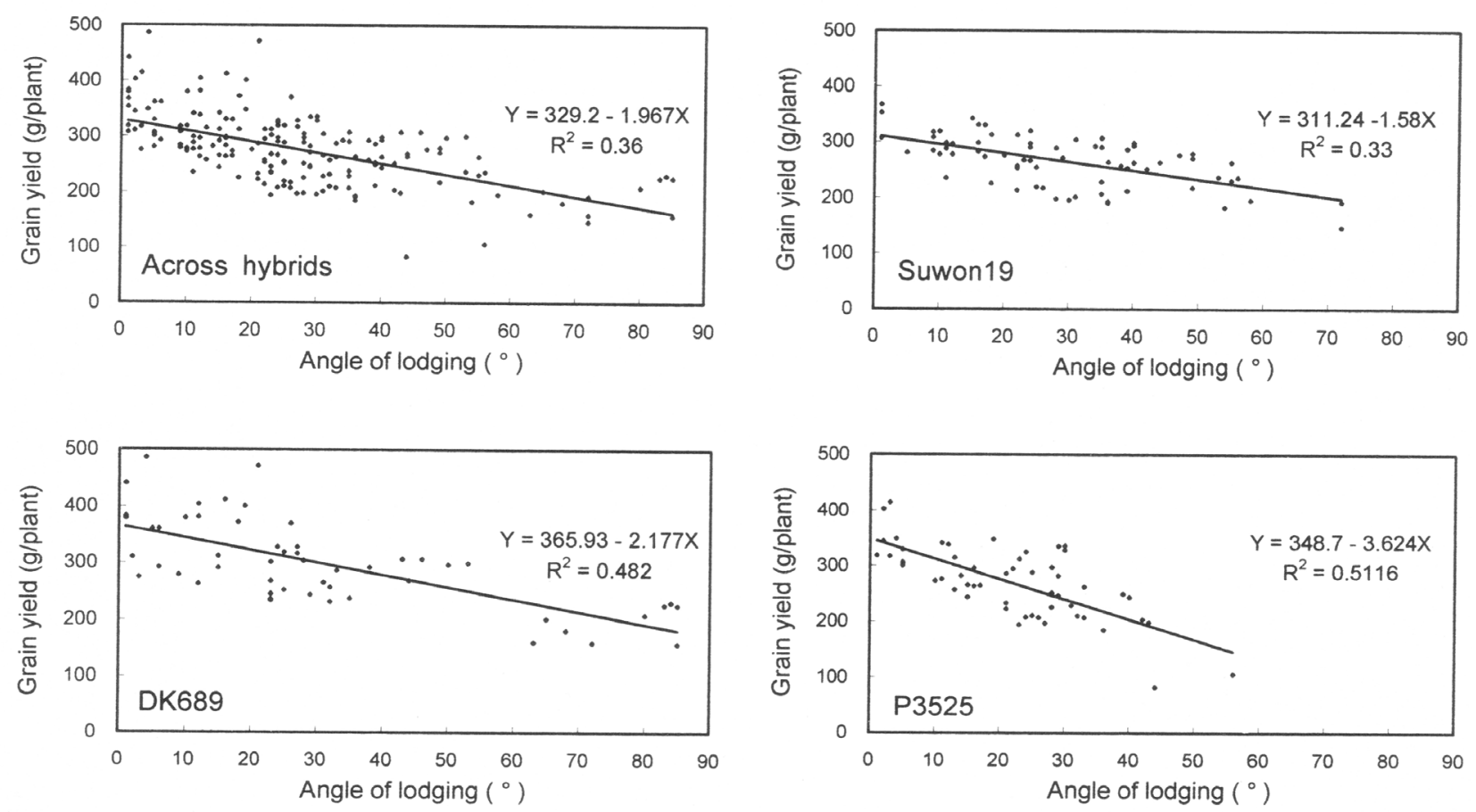

Fig. 1. Regression of grain yield (Y) on angle of lodging (X) for three maize hybrids. 


\section{ACKNOWLEDGMENT}

The first author appreciates the time and effort put together by a junior colleague, So-Jung Kim for data collection and dedicates this report to her. She died of colon cancer in her 20 s.

\section{REFERENCES}

Carter PR and Hudelson KD. 1988. Influence of simulated wind lodging on corn growth and grain yield. J. Prod. Agric. 1:295-299.

Fouéré A, Pellerin S and Duparque A. 1995. A portable electronic device for evaluating root lodging ersistance in maiaze. Agoron. J. 87:1020-1024.

Hebert Y, Barrire Y and Bertholeau JC. 1992. Root lodging resistance in forage maize: Genetic variability of root system and aerial part. Maydica 37:173-183.
Kim SK, Ham YS, Park KY, Park SU, Moon HG, Choi HO and Brewbaker JL. 1978. Disease, insect and lodging resistance super high yielding maize hybrid, "Suwon 19". The Research Report of The rural Development Administration (RDA), Crops 20:149-156.

Liu S, Song F, Liu F, Zhu X and Xu H. 2012. Effect of planting density on root lodging resistance and its relationship to nodal root growth characteristics in maize (Zea mays L.). J. Agri. Sci. 4(12):182-189.

Melchinger AE, Geiger HH and Schmidt GA. 1986. Vertical root-pull resistance and its relationship to root lodging and forage traits in early maturing European inbred line and $\mathrm{F}_{1}$ hybrids of maize. Maydica 31:335-348.

Minami M and Ujihara A. 1991. Effects of lodging on dry matter production, grain yield and nutritional composition at different stages in maize. Japan. J. Crop Sci. 60(1): 107-115.

Stamp P and Kiel C. 1992. Root morphology of maize and its relationship to root lodging. J. Agron. Crop Sci. 168:113118. 